[6] Rombusch, U.K., H. Giesen: Neue Mollier-i, lg p-Diagramme für die Kältemittel R11, R12, R13 und R21. Kältetechnik - Klimatisierung, 18. Jg. (1966) H. 2.

[7] Taniguchi, H., K. Kudo, W.H. Giedt, I. Park, S. Kumazawa: Analytical and Experimental Investigation of TwoPhase Flow Screw Expanders for Power Generation. ASME Journal of Engineering for Gas Turbines and Power, Vol. 110 (1988) No. 4.

[8] Wittig, S., G. Hinsenkamp: Betriebsverhalten und Kennfeldermittlung einer trockenlaufenden Schrauben-Expan- sionsmaschine im organischen Dampfkreislauf (ORC). Abschlußbericht BMFT-Fkz, 03-2871-3A, Karlsruhe (1989).

[9] Zahoransky, R., P. Knöringer, U. Schelling, S. Wittig: Anmerkungen zum Einsatz und Wirkungsgrad kleiner Kraftmaschinen - Turbinen, Dampfmotor und Schraubenexpander. Wärme, Vol. 91 (1985) Nr. 4.

Eingegangen am 2. Januar 1992

F 4089

\title{
VERANSTALTUNGEN
}

\section{ECLAT '92 in Göttingen}

Die vierte ECLAT wird nach 6 Jahren wieder von der Deutschen Gesellschaft für Materialkunde organisiert, und zwar vom 12. bis 15. Oktober in Göttingen. Dabei stehen Laser-Anwendungen im Mittelpunkt, bei denen Werkstoffe eine besondere Rolle spielen. In den vergangenen sechs Jahren haben wesentliche Fortschritte auf dem Gebiet der Laser, der Sensoren sowie der Handling-Systeme stattgefunden. Dadurch wurden ganz neue Möglichkeiten geschaffen, auch komplexe Werkstoffprobleme im Bereich Lasertechnik anzugehen und dabei reproduzierbare Werkstoffeigenschaften zu erzeugen.

Bei der ECLAT '92 werden alle wissenschaftlichen und technischen Aspekte der Laseranwendung in der Werkstofftechnik behandelt. Ziel dabei ist, industriellen Anwendern die vielfältigen Möglichkeiten und Vorteile der Integration von Lasern in den Produktionsprozeß zu verdeutlichen.

Im einzelnen werden behandelt:

- ProzeB-Modellierung/Expertensysteme,

- neue Laserquellen/neue Verfahren,

- Lithographie, Oberflächen-Prägung,

- Laser-unterstützte PVD/CVD-Dünnschichttechnologie,

- Oberflächenbehandlung.
- Gefügeaspekte, Beanspruchungseigenschaften, Eigenspannungen, Ermüdung,

- Einsatz von nichtmetallischen Werkstoffen,

- Laser-gerechte Konstruktion und Produktion,

- Qualitätssicherung.

Fachleute aus allen technischen Sparten, in denen Lasertechnik eine Rolle spielen könnte, aus Europa und Übersee werden erwartet. Tagungssprachen sind Englisch und Deutsch mit Simultanübersetzung in beide Sprachen.

Mit der Tagung verbunden ist eine Ausstellung von Geräten und Zubehör.

Das Programm steht ab Juni 1992 zur Verfügung und kann angefordert werden bei der Deutschen Gesellschaft für Materialkunde e.V., Adenauerallee 21, D-6370 Oberursel, Tel. : 06171/4081.

F 4001

21. ICLP - Internationale Blitzschutzkonferenz

21st International Conference on Lightning Protection (ICLP)

Die 21. Internationale Blitzschutzkonferenz veranstaltet der Ausschuß für Blitzschutz und Blitzforschung im Ver- band Deutscher Elektrotechniker (VDE) vom 22. bis 25. September 1992 im Berliner Kongreßzentrum ICC. Ziel der Konferenz ist es, die neuesten wissenschaftlichen Erkenntnisse und praktischen Erfahrungen in der Blitzschutztechnik vorzustellen und zu diskutieren. Angesprochen sind Wissenschaftler wie auch Konstrukteure und Anwender von Blitzschutzsystemen.

Die Themenkreise:

- Blitzentladung,

- Blitzeinschlag,

- Ableitung des Blitzstromes und Erdung,

- elektromagnetischer Blitzimpuls (LEMP) und Induktionswirkungen,

- Rechentechnische und experimentelle Simulation von Blitzwirkungen,

- Schutz elektronischer Geräte und Systeme,

- praktische Probleme des Blitzschutzes.

Parallel werden in einer Ausstellung aktuelle Blitzschutztechniken, relevante Meß- und Prüftechnik wie auch geeignete Software gezeigt.

Anmeldung und Information bei der VDE-Zentralstelle Tagungen und Seminare, Stresemannallee 15, 6000 Frankfurt a.M. 70, Tel. : 069/6308-321/202.

F 4002

\section{NACHRICHTEN}

Ausschreibung: Internationaler Preis der Graphischen Datenverarbeitung '92

Wie bereits in den vergangenen Jahren schreibt die Dr. Jürgen Schneider Stiftung, gemeinnützige Stiftung zur Förderung des Technologie-Transfers, Darmstadt, in Zusammenarbeit mit der Eurographics, Genf, und der Gesellschaft für Informatik, Bonn, auch in diesem Frühjahr wieder ihren ,Internationalen Preis der Graphischen Datenverarbeitung" aus.
Um den mit insgesamt über 50000,DM dotierten Stiftungspreis können sich Personen und Personengruppen bewerben, die sich in Praxis und Forschung mit Graphischer Datenverarbeitung beschäftigen. Die eingereichten Arbeiten, die bislang weder veröffentlicht noch prämiert sein dürfen, müssen sich schwerpunktmäßig mit Problemkreisen der Entwicklung und/oder Optimierung von Hard- und Softwaremodulen für die Graphische Datenverarbeitung befassen und dürfen nicht älter als zwei Jahre sein. Zugelassen sind darüber hinaus projektorientierte Machbarkeitsstudien und Wirtschaftlichkeitsanalysen.

Einsendeschluß ist der 30. Juni 1992. Die Preisverleihung, über die eine unabhängige internationale Fachjury entscheidet, findet im November 1992 statt.

Weitere Informationen gibt das Stiftungs-Sekretariat: Dr. Jürgen Schneider Stiftung, Gemeinnützige Stiftung zur Förderung des Technologie-Transfers, $z$. Hd. Frau Cornelia Steiner, Osannstraße 11, 6100 Darmstadt, Tel.: 06151/ 425272 .

F 4003 